\title{
Spatial Encoding Using a Code Division Technique for Fast Ultrasound Imaging
}

\section{Gran, Fredrik; Jensen, Jørgen Arendt}

\section{Published in:}

I E E E Transactions on Ultrasonics, Ferroelectrics and Frequency Control

Link to article, DOI:

10.1109/TUFFC.2008.613

Publication date:

2008

Document Version

Publisher's PDF, also known as Version of record

Link back to DTU Orbit

Citation (APA):

Gran, F., \& Jensen, J. A. (2008). Spatial Encoding Using a Code Division Technique for Fast Ultrasound Imaging. I E E E Transactions on Ultrasonics, Ferroelectrics and Frequency Control, 55(1), 12-23. https://doi.org/10.1109/TUFFC.2008.613

\section{General rights}

Copyright and moral rights for the publications made accessible in the public portal are retained by the authors and/or other copyright owners and it is a condition of accessing publications that users recognise and abide by the legal requirements associated with these rights.

- Users may download and print one copy of any publication from the public portal for the purpose of private study or research.

- You may not further distribute the material or use it for any profit-making activity or commercial gain

- You may freely distribute the URL identifying the publication in the public portal 


\title{
Spatial Encoding Using a Code Division Technique for Fast Ultrasound Imaging
}

\author{
Fredrik Gran and Jørgen Arendt Jensen, Senior Member, IEEE
}

\begin{abstract}
This paper describes a method for spatial encoding in synthetic transmit aperture ultrasound imaging. This allows several ultrasonic sources to be active simultaneously. The method is based on transmitting pseudorandom sequences to spatially encode the transmitters. The data can be decoded after only one transmission using the knowledge of the transmitted code sequences as opposed to other spatial encoding techniques, such as Hadamard or Golay encoding. This makes the method less sensitive to motion, and data can be acquired using fewer transmissions. The aim of this paper is to analyze the underlying theory and to test the feasibility in a physical system. The method has been evaluated in simulations using Field II in which the point-spread functions were simulated for different depths for a $7 \mathrm{MHz}$ linear array transducer. A signal-to-noise ratio (SNR) simulation also was included in the study in which an improvement in SNR of $\sim 1.5 \mathrm{~dB}$ was attained compared to the standard synthetic transmit aperture (STA) firing scheme. Considering the amount of energy transmitted, this value is low. A plausible explanation is given that is verified in simulation. The method also was tested in an experimental ultrasound scanner and compared to a synthetic transmit aperture ultrasound imaging scheme using a sinusoidal excitation. The performance of the proposed method was comparable to the reference with respect to axial and lateral resolution, but it displayed poorer contrast with sidelobe levels at $\sim-40 \mathrm{~dB}$ compared to the mainlobe.
\end{abstract}

\section{INTRODUCTION}

A CONVEntional ultrasound image consists of a number of lines. A line is measured by a focused transmission in a given direction, and the received signals can be beamformed using either fixed or dynamic focusing. If dynamic focusing in transmit is desirable, several transmissions have to be made per image line. To form a complete, high-quality image, a large number of transmissions have to be made that imposes a severe restriction on stationarity.

In synthetic transmit aperture (STA) ultrasound imaging [1], [2], one transmitting element is active in every transmission, and one or several receiving elements are actively listening for echoes only from this transmitting element. Because only one element is active in every transmission, the received echoes resulting from that transmission

Manuscript received May 12, 2005; accepted August 26, 2007. This work was supported by grant 9700883, 9700563 and 26-01-0178 from the Danish Science Foundation and by B-K Medical A/S, Denmark.

The authors are with the Center for Fast Ultrasound Imaging, Ørsted•DTU, Technical University of Denmark, 2800 Kgs. Lyngby Denmark (e-mail: fg@oersted.dtu.dk).

Digital Object Identifier 10.1109/TUFFC.2008.613 can be beamformed with dynamic receive focusing to yield several in-parallel created receive lines. A larger transmit aperture can be synthesized by changing the physical location of the active element in the next transmission, beamforming the echoes, and combining the results from the two transmissions. This assumes that the object under investigation is fully stationary. The approach can be extended to cover a large amount of transmissions as long as this condition is not violated. Therefore, STA provides means for both dynamic transmit and receive focusing with a faster acquisition rate because several receive lines are created in parallel.

Two problems arise associated with the STA method.

- Motion leads to noncoherent summation of the data from different transmissions.

- The use of only one transducer element results in poor signal-to-noise ratio (SNR).

The use of virtual sources [3]-[6] has been suggested to increase the SNR. This method relies on using a focused subarray and treats the focus point as a virtual transmitting element. In [7]-[11] the use of linear frequency modulated signals (chirps) was suggested to increase the SNR. These signals make it possible to transmit longer signals with more energy by increasing (or decreasing) the instantaneous frequency of the signal linearly. By matched (or mismatched) filtration, the same axial resolution as with a short excitation waveform can be achieved. A combination of the two approaches can give a significant increase in penetration depth [12].

Spatial encoding has been suggested as a means of increasing the SNR. By spatial encoding, several transmitters $^{1}$ can be excited simultaneously and separated at the receiver. In this way, more acoustic energy can be transmitted with an increase in SNR as a result. Chiao et al. [13] and later Misaridis and Jensen [14] suggested the use of Hadamard encoding to spatially encode the transmitters. The excitation waveforms on the individual transmitters are multiplied by the coefficients of a row (or column) of the Hadamard matrix of the same dimension as the number of transmitters. The decoding is done by adding and subtracting the echoes from transmissions that have been premultiplied by different rows of the Hadamard matrix. Naturally, the same number of transmissions have to be made as the number of active transmitters before the echoes can be decoded. This imposes a restriction on the object under investigation to be fully stationary for

\footnotetext{
${ }^{1}$ The term transmitter can represent either a single transducer element or virtual source.
} 
the decoding to be done without any artifacts. Chiao and Thomas [15] suggested another method for spatially encoding the transmitters using Golay codes. This method, however, also requires the same number of transmissions as number of active transmitters and suffers from the same drawbacks as Hadamard encoding.

Yet another method for spatial encoding was suggested in [16] and was further developed in [17]. The method is based on dividing the available transducer bandwidth into several subbands with ideally disjoint spectral support. In every transmission, each transmitter is assigned an individual band. Therefore, the information from a specific transmitter can be separated from the other transmitters instantaneously at the receiver with a simple filtering operation. However, to cover the full bandwidth with all transmitters, several transmissions have to be done to synthesize a broad band spectrum.

A problem for all these approaches, however, is that the decoding is based on data acquired from several transmissions. This issue is addressed in this paper. The purpose is to investigate a method for spatial encoding in which the different signals can be decoded with data from one single transmission. A method for spatial encoding based on the findings in [18], [19] using pseudo-random sequences is proposed. Full bandwidth decoding can be performed instantaneously at the receiver using only one transmission. Pseudo-random codes were suggested in [20], [21] for spatial encoding purposes. The received signal was modeled as the weighted sum of the system impulse response for certain scatterer positions. A fixed grid of scatterers is defined, and the weights (scatterer strengths) in the image are estimated. In this paper, however, several transmitters are excited simultaneously and are decoded instantaneously at the receiver by estimating the scattering functions between the transmitters and the receivers. The scattering functions are modeled as finite-impulse response (FIR) filters, and no scatter map is defined, which means that no assumptions on the geometry is made. The decoded echoes then are beamformed using a synthetic transmit aperture focusing technique. The aim of this paper is to explain the theory of the method and to test the feasibility in a physical system.

The paper is organized as follows: in Section II the underlying theory is explained and analyzed, in Section III the beamforming associated with the simulations and measurements is reviewed. Simulations of the point-spread functions (PSF) for different depths are given in Section IV along with an analysis of the (peak) SNR. In Section V the PSF is measured in a water tank. and In Section VI concluding remarks are given.

\section{THEORY}

In this section, the theoretical framework for the approach will be derived.

\section{A. System Model}

Consider a system with $K$ transmitters and $Q$ receivers. The $k$ :th transmitter is transmitting the code sequence $x_{k}(t)$. It is assumed that the system is fully linear and that the interaction between the ultrasonic wave and the medium can be described by a linear process. The contribution from transmitter $k$ of the scattered and received signal on receiver $q$ can be written [22]:

$$
y_{q}^{k}(t)=\left\{\sum_{p=0}^{P} s_{p}\left(\vec{r}_{p}\right) h_{e}\left(\vec{r}_{k}, \vec{r}_{p}, t\right) \star h_{r}\left(\vec{r}_{p}, \vec{r}_{q}, t\right)\right\} \star x_{k}(t)
$$

where $P$ is the total number of scatters in the medium (may be infinite), and $s_{p}\left(\vec{r}_{p}\right)$ is the scatter strength of the $p$ :th scatterer. $h_{e}\left(\vec{r}_{k}, \vec{r}_{p}, t\right)$ is the spatial impulse response from the $k$ :th transmitter to the $p$ :th scatterer, $h_{r}\left(\vec{r}_{p}, \vec{r}_{q}, t\right)$ is the spatial impulse response from the scatterer to the $q$ :th receiver, and $\star$ denotes the convolution operator in the time domain. $\vec{r}_{p}$ is the position of the $p$ :th scatterer, $\vec{r}_{k}$ is the position of the $k$ :th transmitter and $\vec{r}_{q}$ is the position of the $q$ :th receiver. The spatial impulse response represents all phenomena that affects the acoustic wave, such as the electromechanical impulse response of the transducer and attenuation. A scattering function between the $k$ :th transmitter and the $q$ :th receiver is now defined as:

$$
h_{k q}(t)=\sum_{p=0}^{P} s_{p}\left(\vec{r}_{p}\right) h_{e}\left(\vec{r}_{k}, \vec{r}_{p}, t\right) \star h_{r}\left(\vec{r}_{p}, \vec{r}_{q}, t\right) .
$$

Note that the spatial dependence of this function has been totally incorporated in the subindexes $k q$. The total received signal on the $q$ :th receiver now can be written as:

$$
y_{q}(t)=\sum_{k=1}^{K} h_{k q}(t) \star x_{k}(t)
$$

This signal is digitized for the purpose of applying digital signal processing. Now, the digitized, received signal under influence of noise is written:

$$
y_{q}(n)=\sum_{k=1}^{K} h_{k q}(n) \star x_{k}(n)+v_{q}(n),
$$

where $v_{q}(n)$ is the digitized noise process on the $q$ :th receiver, and it is assumed to be a zero mean and Gaussian distributed process. $h_{k q}(n)$ is the digitized version of the scattering function $h_{k q}(t)$ and $x_{k}(n)$ is the digitized version of $x_{k}(t)$ with a duration of $N$ samples. The attenuation in the medium makes the received signal decay over time [23]. Therefore, it is reasonable to assume that the scattering function is well modeled as a (possibly long) FIR process, such that the transfer function of the scattering function can be written:

$$
H_{k q}\left(z^{-1)}=\sum_{m=0}^{M-1} h_{k q}(m) z^{-m}\right.
$$




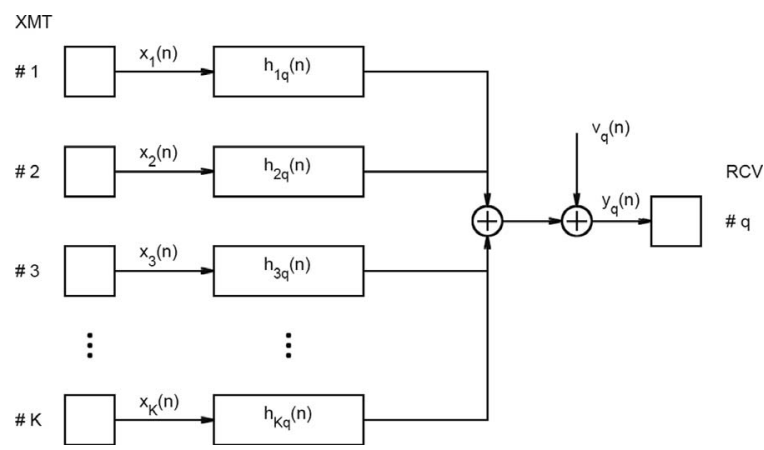

Fig. 1. The network structure of the input/output model of (6).

where $M$ is the length of the impulse responses and $z^{-1}$ is the unit backward-shift operator. The output at the $q$ :th receiver can be written:

$$
y_{q}(n)=\sum_{k=1}^{K} \sum_{m=0}^{M-1} h_{k q}(m) x_{k}(n-m)+v_{q}(n) .
$$

The network structure of (6) can be seen in Fig. 1.

\section{B. Decoding and Separation by Estimation}

The sampled received signal in (6) is now written as a column vector: $y_{q}=\left(y_{q}(0) y_{q}(1) \ldots y_{q}(N+M-2)\right)^{T}$, which implies that the convolution between the transmitted waveform and the corresponding scattering function can be written as a matrix operation:

$$
\mathbf{y}_{q}=\sum_{k=1}^{K} \mathbf{X}_{k} \mathbf{h}_{k q}+\mathbf{v}_{q}
$$

where:

$$
\mathbf{h}_{k q}=\left(h_{k q}(0) h_{k q}(1) \ldots h_{k q}(M-1)\right)^{T}
$$

and:

$$
\mathbf{X}_{k}=\left(\begin{array}{cccc}
x_{k}(0) & 0 & \ldots & 0 \\
x_{k}(1) & x_{k}(0) & \ddots & 0 \\
\vdots & \ddots & \ddots & \vdots \\
x_{k}(N-1) & \ddots & \ddots & x_{k}(0) \\
0 & x_{k}(N-1) & \ddots & x_{k}(1) \\
\vdots & \ddots & \ddots & \vdots \\
0 & 0 & \ldots & x_{k}(N-1)
\end{array}\right) .
$$

and $\mathbf{v}_{q}$ is a zero mean noise process with Gaussian probability distribution and autocovariance matrix $E\left[\mathbf{v}_{q} \mathbf{v}_{q}^{T}\right]=$ $\mathbf{Q}_{v}{ }^{2}$. The result in (7) can be written using a more compact notation by grouping the signal matrices $\mathbf{X}_{k}$ and the scattering function vectors $\mathbf{h}_{k q}$ :

\footnotetext{
${ }^{2} \mathbf{A}^{T}$ denotes the transpose of $\mathbf{A}$ and $E[\mathbf{Q}]$ denotes the expectancy value of $\mathbf{Q}$.
}

$$
\mathbf{y}_{q}=\underbrace{\left(\begin{array}{llll}
\mathbf{X}_{1} & \mathbf{X}_{2} & \ldots & \mathbf{X}_{K}
\end{array}\right)}_{\mathbf{X}} \underbrace{\left(\begin{array}{c}
\mathbf{h}_{1 q} \\
\mathbf{h}_{2 q} \\
\vdots \\
\mathbf{h}_{K q}
\end{array}\right)}_{\mathbf{h}_{q}}+\mathbf{v}_{q} .
$$

The maximum likelihood estimate of the scattering function vector $\mathbf{h}_{q}$ in (10) is found by first defining the probability distribution of receiving the output $\mathbf{y}$ given a set of scattering functions. The probability distribution under the assumption that the scattering function vector is given only depends on the noise characteristics because this is the only part that is stochastic. Because the noise is assumed Gaussian distributed, the probability distribution is given by:

$$
\begin{aligned}
p_{\mathbf{y}_{q} \mid \mathbf{h}_{q}}\left(\mathbf{y}_{q} \mid \mathbf{h}_{q}\right)=\frac{1}{\sqrt{(2 \pi)^{N+M-1} \operatorname{det}\left(\mathbf{Q}_{v}\right)}} \\
\times \exp \left(-\frac{1}{2}\left(\mathbf{y}_{q}-\mathbf{X} \mathbf{h}_{q}\right)^{T} \mathbf{Q}_{v}^{-1}\left(\mathbf{y}_{q}-\mathbf{X} \mathbf{h}_{q}\right)\right) .
\end{aligned}
$$

The objective is to find the set of scattering functions $\mathbf{h}_{q}$ that maximizes the probability distribution in (11), such that:

$$
\hat{\mathbf{h}}_{q}=\arg \max _{\mathbf{h}_{q}} p_{\mathbf{y}_{q} \mid \mathbf{h}_{q}}\left(\mathbf{y}_{q} \mid \mathbf{h}_{q}\right)
$$

The solution to this well-known optimization problem is given by [24]:

$$
\left(\mathbf{X}^{T} \mathbf{Q}_{v}^{-1} \mathbf{X}\right) \hat{\mathbf{h}}_{q}=\mathbf{X}^{T} \mathbf{Q}_{v}^{-1} \mathbf{y}_{q}
$$

The solution to (13) is especially tractable when the noise process is white with variance $\sigma_{v}^{2}$, such that $\mathbf{Q}_{v}=\sigma_{v}^{2} \mathbf{I}$, where $\mathbf{I}$ is the identity matrix. The linear system of equations in (13) then is reduced to:

$$
\left(\mathbf{X}^{T} \mathbf{X}\right) \hat{\mathbf{h}}_{q}=\mathbf{X}^{T} \mathbf{y}_{q}
$$

Further, the MISO (multiple-input-single-output) model in (10) can be generalized to the MIMO (multiple-inputmultiple-output) case by writing:

$$
\underbrace{\left(\begin{array}{c}
\mathbf{y}_{1}^{T} \\
\mathbf{y}_{2}^{T} \\
\vdots \\
\mathbf{y}_{Q}^{T}
\end{array}\right)}_{\mathbf{Y}}=\underbrace{\left(\begin{array}{c}
\mathbf{h}_{1}^{T} \\
\mathbf{h}_{2}^{T} \\
\vdots \\
\mathbf{h}_{Q}^{T}
\end{array}\right)}_{\mathbf{H}} \mathbf{X}^{T}+\underbrace{\left(\begin{array}{c}
\mathbf{v}_{1}^{T} \\
\mathbf{v}_{2}^{T} \\
\vdots \\
\mathbf{v}_{Q}^{T}
\end{array}\right)}_{\mathbf{V}},
$$

where each column in the matrix $\mathbf{Y}$ represents one (multidimensional) output sample of the receiving array ( $Q$ receiving transducer elements). The set of linear equations corresponding to (14) is for the MIMO case found to be:

$$
\hat{\mathbf{H}}\left(\mathbf{X}^{T} \mathbf{X}\right)=\mathbf{Y X}
$$




\section{Identifiability}

The solution to both (14) and (16) are uniquely defined if and only if $\mathbf{X}$ has full column rank [25]. This means that the system of equations must not be under-determined. By analyzing the dimensions of the matrix $\mathbf{X}$, a lower bound on the code length can be derived. The dimensions of $\mathbf{X}$ are $M+N-1 \times K M$, imply that:

$$
\begin{aligned}
M+N-1 & \geq K M \\
\Downarrow & \\
N & \geq(K-1) M+1 .
\end{aligned}
$$

The minimum code length, thus, is dependent on the number of active transmitters in every transmission and the length of the scattering functions that should be estimated.

\section{Estimation Error}

The derivation of the mean square estimation error follows the derivation in [26]. The model is given in (10), and the noise is assumed white with zero mean. If $\mathbf{X}$ has full rank, the maximum likelihood estimate of $\mathbf{h}_{q}$ can be written:

$$
\begin{aligned}
\hat{\mathbf{h}}_{q} & =\left(\mathbf{X}^{T} \mathbf{X}\right)^{-1} \mathbf{X}^{T} \mathbf{y}_{q} \\
& =\underbrace{\left(\mathbf{X}^{T} \mathbf{X}\right)^{-1} \mathbf{X}^{T} \mathbf{X} \mathbf{h}_{q}}_{\mathbf{h}_{q}}+\underbrace{\left(\mathbf{X}^{T} \mathbf{X}\right)^{-1} \mathbf{X}^{T} \mathbf{v}_{q}}_{\mathbf{e}_{q}},
\end{aligned}
$$

where $\mathbf{e}_{q}$ is the estimation error on the $q$ :th receiving transducer element. The covariance matrix of the estimation error is defined as:

$$
\begin{aligned}
E\left[\mathbf{e}_{q} \mathbf{e}_{q}^{T}\right] & =E\left[\left(\mathbf{X}^{T} \mathbf{X}\right)^{-1} \mathbf{X}^{T} \mathbf{v}_{q} \mathbf{v}_{q}^{T} \mathbf{X}\left(\mathbf{X}^{T} \mathbf{X}\right)^{-1}\right] \\
& =\left(\mathbf{X}^{T} \mathbf{X}\right)^{-1} \mathbf{X}^{T} E\left[\mathbf{v}_{q} \mathbf{v}_{q}^{T}\right] \mathbf{X}\left(\mathbf{X}^{T} \mathbf{X}\right)^{-1} \\
& =\left(\mathbf{X}^{T} \mathbf{X}\right)^{-1} \mathbf{X}^{T} \mathbf{Q}_{v} \mathbf{X}\left(\mathbf{X}^{T} \mathbf{X}\right)^{-1} \\
& =\left(\mathbf{X}^{T} \mathbf{X}\right)^{-1} \sigma_{v}^{2}
\end{aligned}
$$

The mean square error of the estimate for the $m$ :th sample in a given scattering function $\left\{h_{k q}(m)\right\}_{m=0}^{M-1}$, therefore, is given by:

$$
\begin{aligned}
E\left[\mid h_{k q}(m)-\right. & \left.\left.\hat{h}_{k q}(m)\right|^{2}\right]= \\
& \sigma_{v}^{2}\left\{\left(\mathbf{X}^{T} \mathbf{X}\right)^{-1}\right\}_{k M-M+m, k M-M+m}
\end{aligned}
$$

This means that the decoded noise component on the $m$ :th sample of the scattering function for the $q$ :th receiver and the $k$ :th transmitter, will be $\sigma_{v}^{2}$ multiplied by diagonal element number $k M-M+m$ of the matrix $\left(\mathbf{X}^{T} \mathbf{X}\right)^{-1}$.

For instance, if the code sequences are completely uncorrelated and have perfect autocorrelation functions (delta functions), the matrix:

$$
\mathbf{X}^{T} \mathbf{X}=N \mathbf{I}
$$

will be diagonal. Therefore:

$$
\left(\mathbf{X}^{T} \mathbf{X}\right)^{-1}=\frac{1}{N} \mathbf{I}
$$

and the decoded noise variance will be improved by a factor of $N$. In this paper, however, the chosen code sequences will not be sufficiently uncorrelated to approximate (21) and (22). Therefore, to analyze the estimation error properly, the full expression in (20) has to be considered.

\section{BEAMFORMING}

This section describes the beamforming that has been applied to properly focus the data. When the data from one specific transmitter is available, either by applying spatial encoding and separating the signals from the different transmitters at the receiver, or using only one transmitter at a time, it is possible to determine where on the aperture the acoustic wave was transmitted, and where it was received. This allows the synthetic aperture focusing technique described in [2] to be applied.

Because the system has access to defocused transmissions from all transmitters on the aperture, it is possible to focus the acoustic energy on any arbitrarily chosen point of interest. The amplitude in a point $p$ in the image is given by:

$$
H\left(\vec{r}_{p}\right)=\sum_{k=1}^{L} \sum_{q=1}^{Q} g_{q}\left(t_{p k q}\right) h_{k q}\left(t_{p k q}\right)
$$

where $g_{q}\left(t_{p k q}\right)$ is a weighting function (apodization) over the receiving aperture that ideally would be changing with spatial coordinates. However, in this paper the apodization is constant over space and is chosen as a Hanning window over all 128 receiving elements. The time signal $h_{k q}(t)$ is the received (and possibly filtered) echo on the $q$ :th receiver, originating from the $k$ :th transmitter. Here $t_{p k q}$ is the time corresponding to the geometrical travel distance from the $k$ :th transmitter to the point $p$ and back to the $q$ :th receiver:

$$
t_{p k q}=\frac{\left\|\vec{r}_{p}-\vec{r}_{k}\right\|+\left\|\vec{r}_{q}-\vec{r}_{p}\right\|}{c},
$$

where $c$ is the speed of sound, and $\vec{r}_{p}-\vec{r}_{k}$ is the vector from the transmitter to the point $p$, and $\vec{r}_{q}-\vec{r}_{p}$ is the vector from the point $p$ to the receiver as indicated in Fig. 2. Summing contributions from all transmissions for all receiving elements gives both dynamic transmit and receive focusing.

\section{Simulations}

A simulation study was conducted to compare the method to conventional STA imaging. For this purpose, the simulation tool Field II [27], [28] was used. The sampling frequency of the simulations was $120 \mathrm{MHz}$. A 7-MHz linear array transducer was simulated. The pitch of the transducer was $0.208 \mathrm{~mm}$, the kerf was $0.035 \mathrm{~mm}$, and the height of the transducer elements was $4.5 \mathrm{~mm}$. The simulated transducer consisted of 128 transducer elements, 


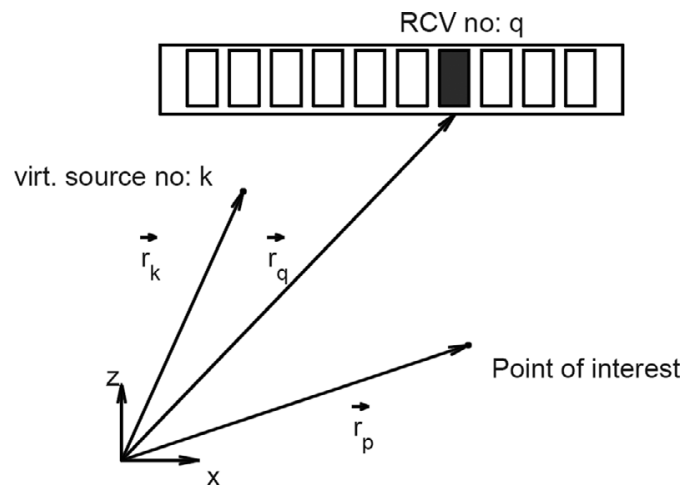

Fig. 2. Principles of synthetic aperture focusing for a point $p$ for the $k$ :th transmitter and the $q$ :th receiver.

and the transducer impulse response was approximated by a two cycle sinusoid at $7 \mathrm{MHz}$ with a temporal Hanning weighting. The transmitting aperture consisted of 64 transmitters for which one transmitter was created by grouping two transducer elements. This was done to correspond to the succeeding experiments in which it is desirable to transmit more acoustic energy to increase SNR. Thus, transmitter one will represent transducer element one and two, transmitter two will represent transducer element three and four, and so on. The spacing between the transmitters thus will be twice the distance between the individual transducer elements. The target was four point scatterers positioned at depths 30, 35, 40, and $45 \mathrm{~mm}$.

\section{A. Reference Simulation}

The reference simulation was created by exciting the transmitters sequentially. Two different simulations were carried out. In the first simulation, a one cycle, $5 \mathrm{MHz}$ sinusoid with a temporal Hanning weighting was used as excitation. In the second simulation, a four cycle, $5 \mathrm{MHz}$ sinusoid with a temporal Hanning weighting was used as excitation. The second simulation was carried out to correspond to the experiments in Section V. The target was four point targets located at depths $30,35,40$, and $45 \mathrm{~mm}$. The received echoes from each transmission were match filtered with the time-reversed version of the excitation waveform, and thereafter, beamformed using the approach given in Section III. The resulting images can be seen as the plots to the left in Figs. 3 and 4.

\section{B. Spatially Encoded Simulation}

The spatially encoded simulation was created by exciting transmitter 1 and 33 in the first transmission, transmitter 2 and 34 in the second transmission, and so on. Thus, two transmitters were active in every transmission. In total 32 transmission were made. To separate the echoes originating from the two different transmitters, two binary code sequences were used. The codes were created from a zero mean Gaussian distributed stochastic process. The code sequence was given the value 1 if the result was greater than zero and -1 if the value was less or equal to zero.

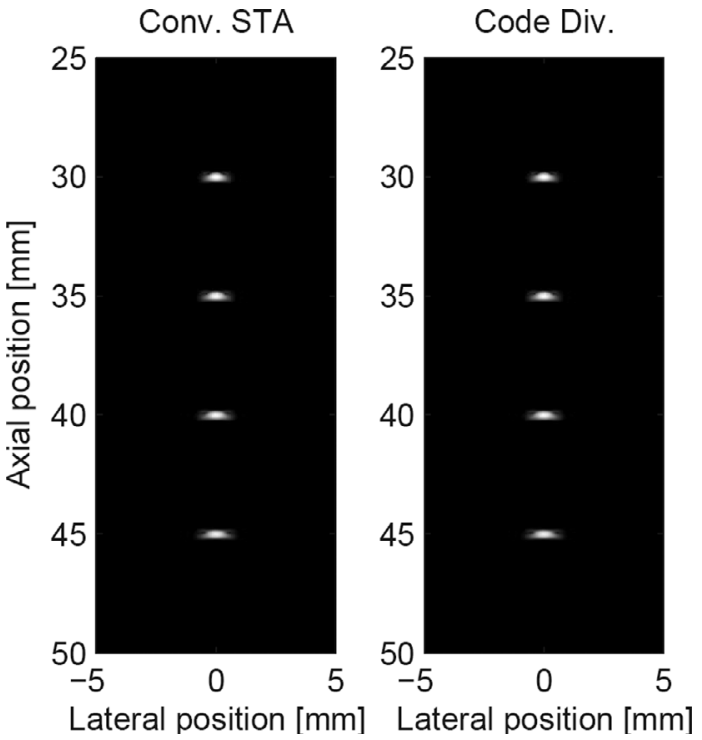

Fig. 3. The PSFs and axial and lateral projections for the two methods when using the one-cycle, $5 \mathrm{MHz}$ chip (left conventional STA, right spatially encoded excitation). The two methods have comparable performance in terms of resolution and contrast. The image obtained using traditional STA used 64 transmissions. The image obtained using spatial encoding used only 32 transmissions. The dynamic range of the $\mathrm{B}$-mode images are $50 \mathrm{~dB}$.

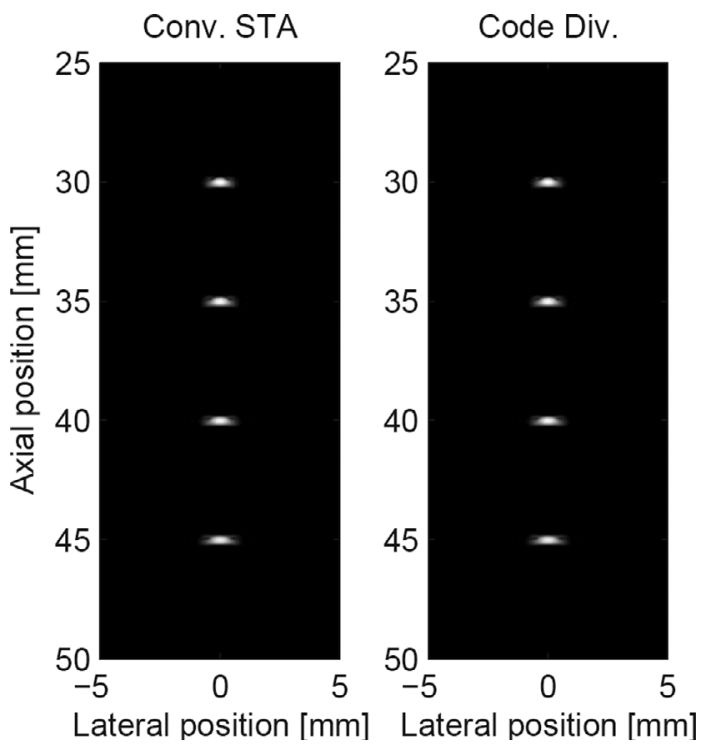

Fig. 4. The PSFs and axial and lateral projections for the two methods when using the four-cycle, $5 \mathrm{MHz}$ chip (left conventional STA, right spatially encoded excitation). The two methods have identical performance in terms of resolution and contrast. The image obtained using traditional STA used 64 transmissions. The image obtained using spatial encoding used only 32 transmissions. The dynamic range of the B-mode images are $50 \mathrm{~dB}$. 

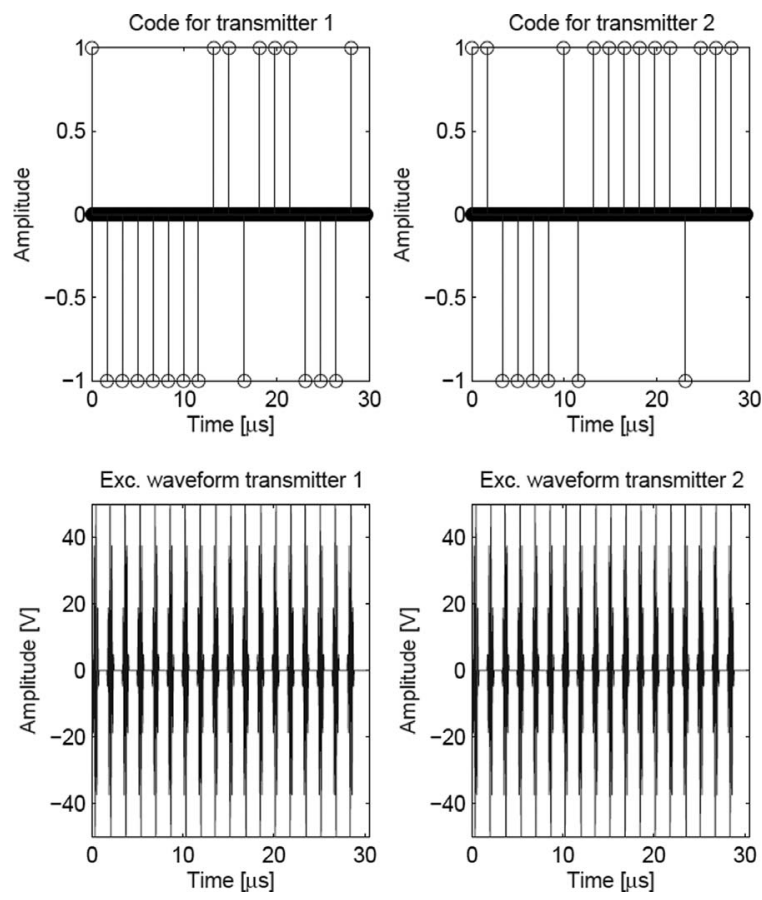

Fig. 5. The binary codes for the two transmitters are given in the top figures. The two lower figures show the actual transmitted waveforms. The coding was generated with a distance between succeeding nonzero samples corresponding to twice the duration of the $c(n)$ waveform.

The duration of the two sequences was 18 bits, and they are denoted $x_{1}(n)$ and $x_{2}(n)$.

Before transmission, the code was oversampled and convolved with a band limited waveform, called a chip waveform. The code is wideband and the purpose of the chip waveform is to reduce the effective bandwidth of the transmitted waveform. Thus, the transmitted waveforms were:

$$
s_{k}(n)=\sum_{l=0}^{N-1} x_{k}(l) c\left(n-l \cdot T f_{s}\right),
$$

where $N$ is the duration of the code sequence, $T$ is the inter chip time interval, $f_{s}$ is the sampling frequency of the ultrasound system, and $c(n)$ is the chip waveform. $T$ was chosen to be $0.8 \mu \mathrm{s}$.

Two simulations were carried out. First, a simulation with a one cycle sinusoid as chip waveform was done, then a simulation using a four-cycle sinusoid was performed. The center frequency of both chip waveforms was $5 \mathrm{MHz}$. The two code sequences and the corresponding two waveforms when using the four-cycle chip waveform can be seen in Fig. 5. The target was four point targets located at depths $30,35,40$, and $45 \mathrm{~mm}$. The signals corresponding to the two transmitters were separated using (14). Thereafter, the separated echoes were match filtered with the time-reversed chip waveform. The beamforming described in Section III was applied, and the PSFs were plotted. The result can be seen as the right images in Fig. 3 (1 cycle chip waveform) and Fig. 4 (four cycle chip waveform). The axial and lateral projections are given in Fig. 6 (broadband)
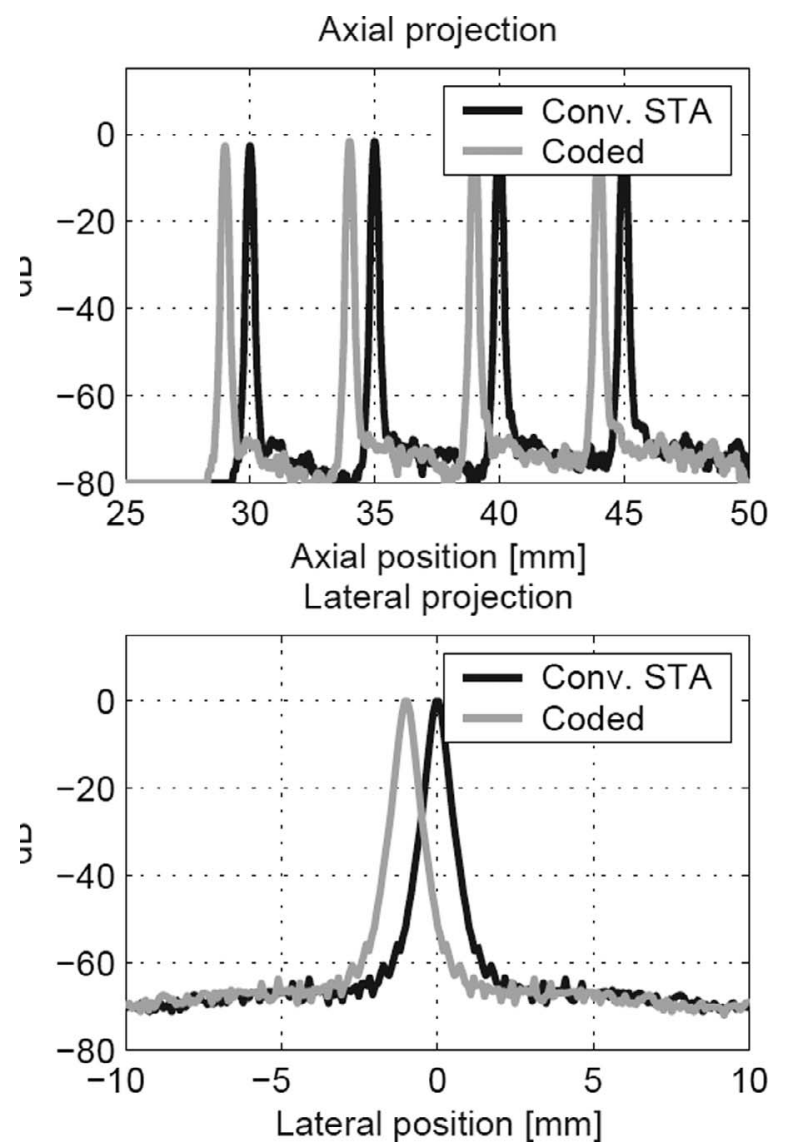

Fig. 6. The axial projections (top plot) and the lateral projections (bottom plot) of the broadband simulation. The reference simulation is given in black, and the coded simulation is given in gray. The coded simulation has been shifted $1 \mathrm{~mm}$ for better visualization. The axial profile of the coded simulation is identical to the reference simulation.

and Fig. 7 (narrow band). The reference simulation is indicated in black and the coded simulation is given in gray. The coded simulations have been shifted $1 \mathrm{~mm}$ to better visualize the curves. It can be seen that the PSFs for the coded method and the reference simulation are the same. This also was expected as the only thing remaining after the decoding in the ideal case (linear propagation and no noise) is the chip waveform. Therefore, the echoes passed to the beamformer for the coded simulation, and the reference simulation will be the same. Consequently, the PSFs will be the same. The axial and lateral resolutions for the broadband simulations are given in Table I, and the resolutions for the narrow band simulations are given in Table II. Because the PSFs for the coded simulations are identical to the reference simulations, only the resolutions for the coded simulations are given.

\section{Signal-to-Noise-Ratio}

When the simulation is done without the influence of noise, the proposed method should find the exact scattering function because the simulation tool is fully linear. However, to evaluate the performance under the influence of noise, a SNR simulation was carried out. The simula- 

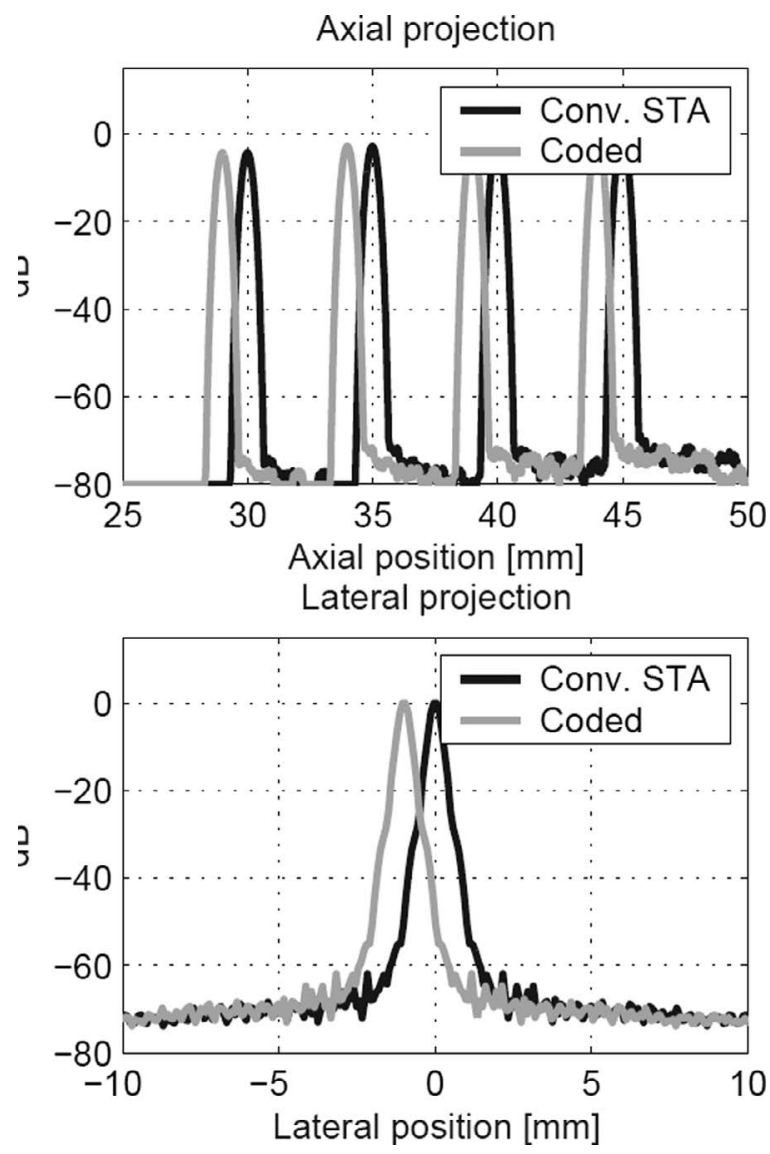

Fig. 7. The axial projections (top plot) and the lateral projections (bottom plot) of the narrowband simulation. The reference simulation is given in black, and the coded simulation is given in gray. The coded simulation has been shifted $1 \mathrm{~mm}$ for better visualization. The axial profile of the coded simulation is identical to the reference simulation.

TABLE I

Resolution as a Function of Depth, Broadband Waveform.

\begin{tabular}{lcccc}
\hline & \multicolumn{2}{c}{ Axial res. mm } & \multicolumn{2}{c}{ Lateral res. mm } \\
\cline { 2 - 5 } Depth & $-3 \mathrm{~dB}$ & $-6 \mathrm{~dB}$ & $-3 \mathrm{~dB}$ & $-6 \mathrm{~dB}$ \\
\hline $30 \mathrm{~mm}$ & 0.109 & 0.163 & 0.119 & 0.251 \\
$35 \mathrm{~mm}$ & 0.109 & 0.160 & 0.149 & 0.285 \\
$40 \mathrm{~mm}$ & 0.107 & 0.160 & 0.185 & 0.326 \\
$45 \mathrm{~mm}$ & 0.107 & 0.158 & 0.218 & 0.361 \\
\hline
\end{tabular}

TABLE II

Resolution as a Function of Depth, Narrowband Waveform.

\begin{tabular}{ccccc}
\hline & \multicolumn{2}{c}{ Axial res. mm } & \multicolumn{2}{c}{ Lateral } \\
\cline { 2 - 5 } Depth. mm \\
\cline { 2 - 5 } & $-3 \mathrm{~dB}$ & $-6 \mathrm{~dB}$ & $-3 \mathrm{~dB}$ & $-6 \mathrm{~dB}$ \\
\hline $30 \mathrm{~mm}$ & 0.300 & 0.430 & 0.170 & 0.306 \\
$35 \mathrm{~mm}$ & 0.301 & 0.430 & 0.221 & 0.360 \\
$40 \mathrm{~mm}$ & 0.300 & 0.430 & 0.251 & 0.425 \\
$45 \mathrm{~mm}$ & 0.300 & 0.429 & 0.295 & 0.458 \\
\hline
\end{tabular}

tions were carried out according to the narrow band simulations in Section IV-A and Section IV-B, but a noise field was added to every set of received data before further processing. The only parameter that separates the coded simulations from the reference simulations is the coding and decoding method. The SNR gain, therefore, will be independent of the choice of chip waveform as long as the reference simulation is carried out with an excitation corresponding to the chip waveform.

The noise fields were generated from a white, zero mean, Gaussian distributed process. By determining the maximum amplitude of the received signals for the spatially encoded approach, the variance of the noise field was adjusted such that a SNR of $20 \mathrm{~dB}$ on the raw channel data was attained. The same variance was used for all transmissions for both the spatially encoded simulation and the reference simulation. The echoes from the spatially encoded simulation were first decoded using the approach described in Section II, then match filtered using the time-reversed version of $c(n)$. The processing of the echoes resulting from the reference simulation involved only matched filtering. The echoes from both simulations were beamformed in exactly the same way. Because the corresponding simulations without the influence of noise were available, the noise component of the images was found by simply subtracting the image influenced by noise by the corresponding image without noise. The (peak) SNR then was evaluated at the positions of the point targets. The noise variance was estimated by choosing an area of $3 \mathrm{~mm} \times 4 \mathrm{~mm}$ around the position of the point in the corresponding noise image:

$$
\begin{aligned}
& \hat{\sigma}^{2}\left(n_{x}, n_{y}\right)= \\
& \frac{1}{N_{x} N_{y}} \sum_{i=n_{x}}^{n_{x}+N_{x}} \sum_{j=n_{y}}^{n_{y}+N_{y}} n^{2}\left(i-\frac{N_{x}}{2}, j-\frac{N_{y}}{2}\right),
\end{aligned}
$$

where $\left(n_{x}, n_{y}\right)$ denotes the point position in samples, $N_{x} \times$ $N_{y}$ is the area over which the variance should be estimated in samples, and $n(i, j)$ is the noise image. The SNR then is estimated by:

$$
\operatorname{SNR}\left(n_{x}, n_{y}\right)=\frac{\left|s\left(n_{x}, n_{y}\right)\right|^{2}}{\hat{\sigma}^{2}\left(n_{x}, n_{y}\right)},
$$

where $s\left(n_{x}, n_{y}\right)$ is the amplitude in the image at the position of the point scatterers. The gain in SNR for the proposed method then was calculated by computing the ratio between the SNR for the spatially encoded approach and the SNR for the approach using a sinusoid excitation. The gain (in $\mathrm{dB}$ ) then is:

$$
G=10 \log _{10}\left(\frac{\mathrm{SNR}_{\text {code.div }}}{\mathrm{SNR}_{\text {pulsed }}}\right) .
$$

The result can be seen in Fig. 8 in which it is compared to the ideal gain in SNR predicted in (20). It can be seen that the the ideal $^{3}$ gain is $\sim 12.5 \mathrm{~dB}$, and the actual gain

\footnotetext{
${ }^{3}$ Ideal gain in this example refers to when the code sequences have perfect autocorrelation functions and are completely uncorrelated.
} 


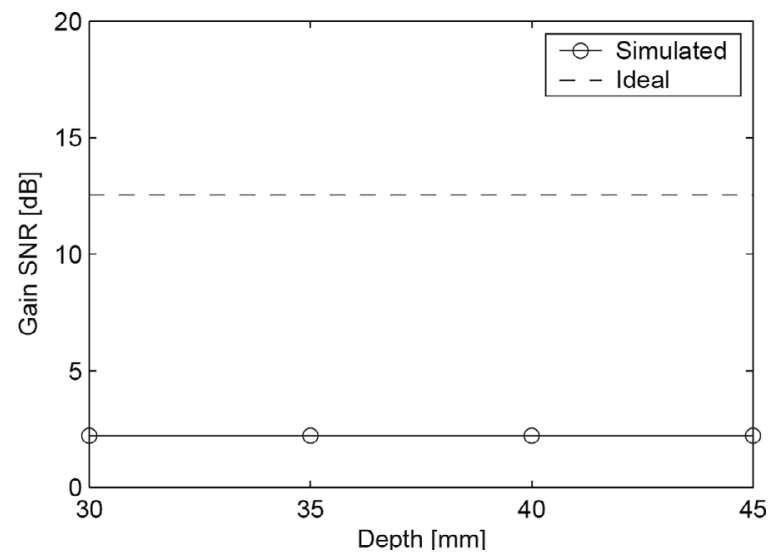

Fig. 8. The SNR as a function of depth. The dashed line indicates the ideal gain in SNR in decibels as predicted in (20). The solid line indicates the simulated result.

is $1.66 \mathrm{~dB}$. It should be noted that the predicted gain in SNR given in (20) was based on the assumption that the matrix $\left(\mathbf{X}^{H} \mathbf{X}\right)^{-1}$ is diagonal. However, in this paper no attempts have been made to make this matrix diagonal. Therefore, the ideal gain in SNR will not be achieved. To evaluate how the estimation process effects the variance of the noise, the diagonal elements of $\left(\mathbf{X}^{H} \mathbf{X}\right)^{-1}$ should be analyzed. In Fig. 9, the diagonal elements are plotted. Each diagonal element corresponds to a specific sample in the impulse response estimate which means that the variance of the estimation error is a function of depth. The estimation error will act as a noise process in the images and scales linearly with the noise variance $\sigma_{v}^{2}$. The average variance of the estimation error can be estimated as:

$$
\tilde{\sigma}_{v}^{2}=\sigma_{v}^{2} \frac{1}{2 M} \sum_{m=1}^{2 M}\left\{\left(\mathbf{X}^{H} \mathbf{X}\right)^{-1}\right\}_{m, m},
$$

and gives an indication of the average noise level after decoding. In this paper for the code sequences chosen, the quantity in (29) will be $\tilde{\sigma}_{v}^{2} \approx 0.73 \sigma_{v}^{2}$, which corresponds to an increase in SNR of approximately $1.4 \mathrm{~dB}$. This also explains the discrepancy between the ideal gain in SNR and the actual gain in SNR simulated in Fig. 8.

\section{Measurements}

This section describes the measurements done to demonstrate the method. The results in Section V-A show the principle performance of the proposed method with two simultaneously transmitting transmitters and a single receiver. In Section V-B, the point spread function is measured and compared to a system using a sinusoidal excitation, sequential excitation, and STA beamforming.

\section{A. Simple Estimation}

The measurements were done using a BK8804 7-MHz, linear array transducer (BK-Medical ApS, Herlev, Copenhagen, Denmark). The experimental ultrasound scanner
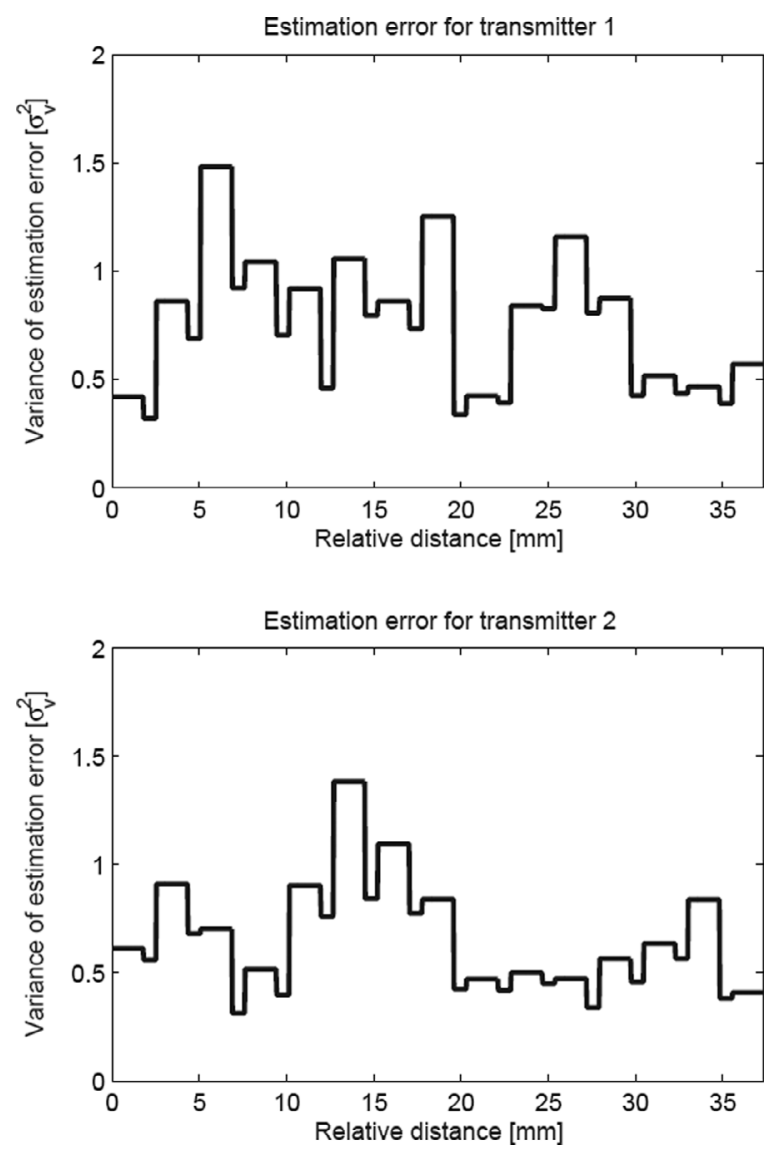

Fig. 9. The variance of the estimation error as a function of depth for the two transmitters. A value above one indicates that the SNR is worsened. A value below one indicates that the SNR is improved compared to the SNR on the raw RF data.

RASMUS [29] was used. RASMUS has access to 128 transmit channels, which implied that the central 128 of the 192 existing transducer elements were used. Throughout Section V-A, transmitter one represents transducer element numbers 43 and 44, and the second transmitter represents element number 63 and 64. Every "virtual" transmitter, thus, is created by two transducer elements. Transducer element number 64 was the active receiver. The experiments were carried out on a string phantom in water. A metal wire was positioned at $42 \mathrm{~mm}$.

1. Reference: The reference was created by exciting each of the transmitters separately and recording the echoes from the metal wire. A four-cycle sinusoid at a center frequency of $5 \mathrm{MHz}$ with temporal Hanning weighting was used as excitation. The center frequency of the excitation waveform was chosen to coincide with the maximum in the system transfer function ${ }^{4}$. The received echoes were match filtered using the time-reversed version of the excitation waveform. The excitation voltage was $\pm 50 \mathrm{~V}$. This experiment was repeated 500 times, and the echoes from the individual experiments were averaged to reduce the noise and give good estimates of the scattering functions

\footnotetext{
${ }^{4}$ This includes both the transducer and the measurement system.
} 

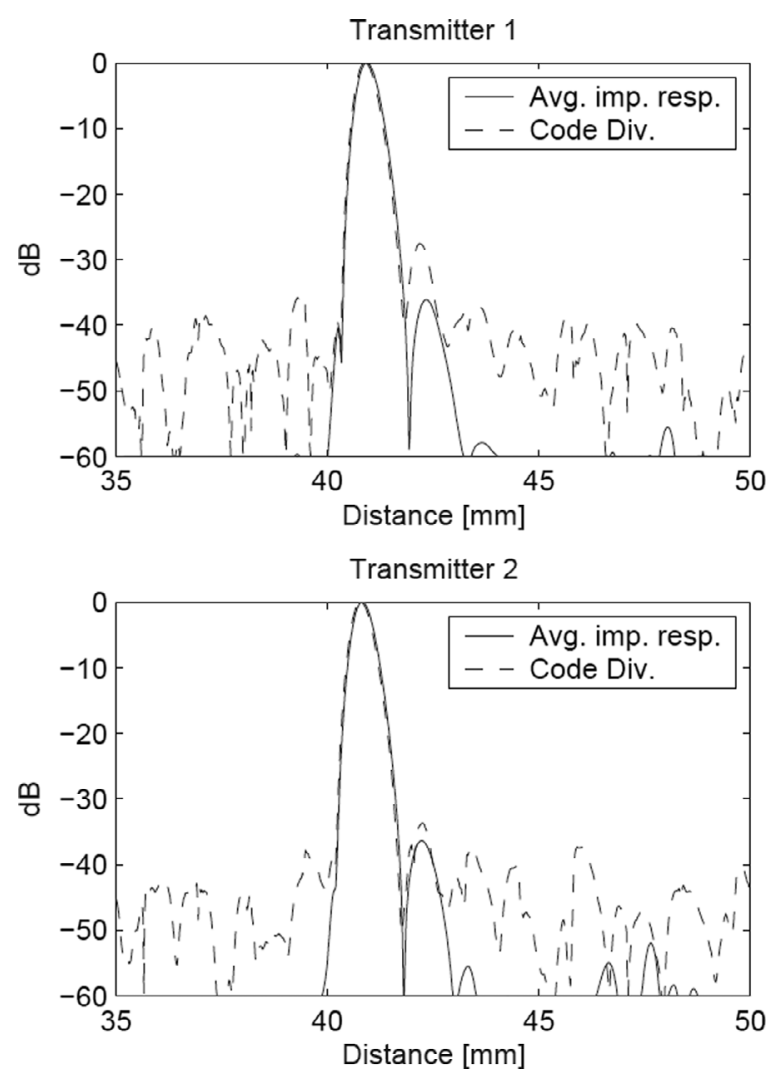

Fig. 10. The envelope of the estimate of the scattering functions for the two transmitters (dashed line). The axial sidelobe level is $\sim 40 \mathrm{~dB}$. Estimates for the true scattering functions were found by exciting the transmitters separately 500 times and calculating the mean profile (solid line).

for this given excitation. The envelope in logarithmic scale can be seen Figs. 10 and 11 as the solid line.

2. Spatially Coded Excitation: The spatially encoded method was tested experimentally using the same code sequences and excitation waveforms as in the narrow band simulations. The transmitters were excited simultaneously, and the echoes from the wire were recorded. The excitation voltage was $\pm 50 \mathrm{~V}$. The estimation procedure given in (14) was used to find the scattering functions corresponding to the two transmitters. The decoded signals then were match filtered with the time reversed version of $c(n)$. The envelope of the result in logarithmic scale can be seen in Figs. 10 and 11 as the dashed line. One realization of the reference experiment is given in Fig. 11 as the dotted line. It can be seen that the coded approach finds the scattering functions with approximately the same accuracy as if the transmitters had been excited separately.

\section{B. Point Spread Function}

The point-spread function was measured using the same wire phantom as before. The same measurement system and transducer was used as in Section V-A. The central 128 transducer elements were used. The transducer elements were grouped in pairs, such that two neighboring
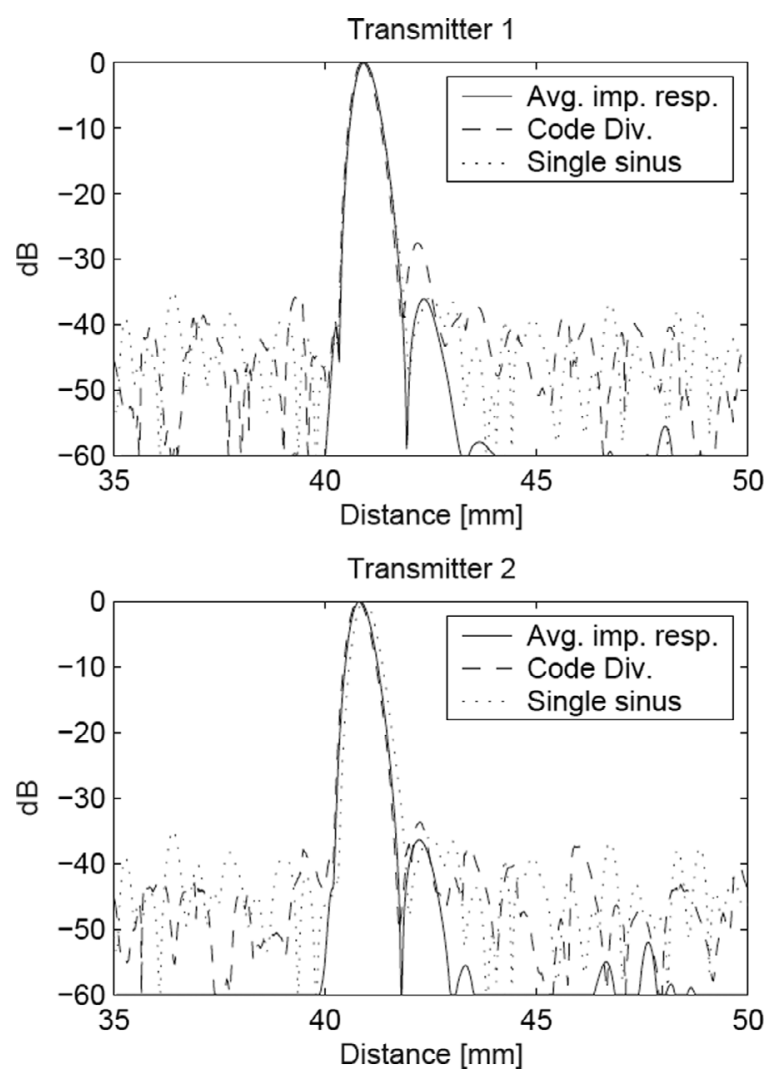

Fig. 11. The envelope of the estimate of the scattering functions for the two transmitters (dashed line). The axial sidelobe level is $\sim 40 \mathrm{~dB}$. The dotted line represents the result from exciting the transmitters sequentially. Estimates for the true scattering functions were found by exciting the transmitters separately 500 times and calculating the mean profile (solid line).

elements emulated one transmitter. Transducer element one and two create transmitter one, and transducer element 65 and 66 create transmitter two. Transmitter three represents element three and four, and transmitter four represents element 67 and 68 , and so on. In total there will be 64 transmitters. All 128 transducer elements were used as the receiving aperture with a Hanning apodization.

1. Reference Experiment: For a reference, the transmitters were excited sequentially using a four cycle sinusoid at $5 \mathrm{MHz}$ with a temporal Hanning weighting. The resulting echoes were match filtered with the time-reversed version of the excitation waveform. Thereafter, they were beamformed using the method described in Section III. The resulting point spread function with a dynamic range of $50 \mathrm{~dB}$ can be seen as the left plot in Fig. 12. The axial and lateral projections were generated by taking the mean of the rows and columns of the envelope data matrix, respectively, and plotting the result.

2. Coded Excitation: The point spread function was obtained by exciting two transmitters simultaneously. In the first transmission transmitter one and two were excited, in the second transmission transmitter three and four were used, and so on until all transmitters had been covered. 

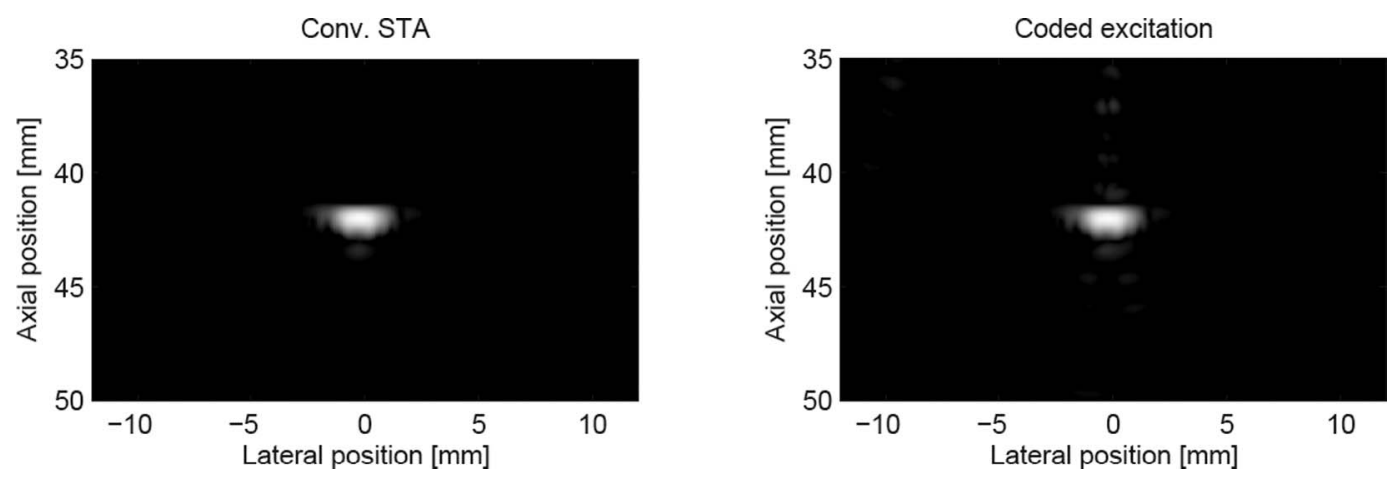

Fig. 12. The PSF of the method based on spatial encoding (right plot), and the PSF of the reference experiment (left plot). The dynamic range of the B-mode images is $50 \mathrm{~dB}$. The spatial encoding approach exhibits slightly poorer axial contrast.

TABLE III

Resolution AT 42 mM.

\begin{tabular}{lcccc}
\hline \multirow{2}{*}{ Depth } & \multicolumn{2}{c}{ Axial res. mm } & \multicolumn{2}{c}{ Lateral res. mm } \\
\cline { 2 - 5 } & $-3 \mathrm{~dB}$ & $-6 \mathrm{~dB}$ & $-3 \mathrm{~dB}$ & $-6 \mathrm{~dB}$ \\
\hline Conv. STA & 0.382 & 0.549 & 0.919 & 1.215 \\
Code Div. & 0.384 & 0.551 & 0.938 & 1.238 \\
\hline
\end{tabular}

The same code sequences and excitation waveforms as in Section V-A were used. The decoding and filtering performed on the received signals also follows the outline of Section V-A. Once the signals had been separated, the beamforming described in Section III was applied. The resulting point spread function with a dynamic range of $50 \mathrm{~dB}$ can be seen as the plot on the right in Fig. 12. The axial and lateral projections in Fig. 13 were generated by taking the mean of the rows and columns of the envelope data matrix, respectively, and plotting the result. The axial sidelobe level of the code division experiment is approximately $-40 \mathrm{~dB}$. The sidelobes results from modeling errors (nonlinearities) and noise. The axial and lateral resolution can be seen in Table III. It can be seen that the resolution is comparable for the two methods. The axial contrast also was approximately the same with slightly poorer contrast in front of the string for the spatially encoded approach.

\section{Conclusions}

In this paper, a method for spatial encoding was proposed. The method is based on a linear approximation, and the signals originating from different transmitters are separated using maximum likelihood estimation. The data can be separated after only one transmission, which shows that fewer transmissions can be made to form a complete image. However, to solve the estimation problem uniquely, a lower limit on the code duration appears that depends on the number of active transmitters and the duration of the scattering functions. According to the theory, in the ideal case, the gain in SNR will be proportional to the energy of the code sequences. This was studied in simu-
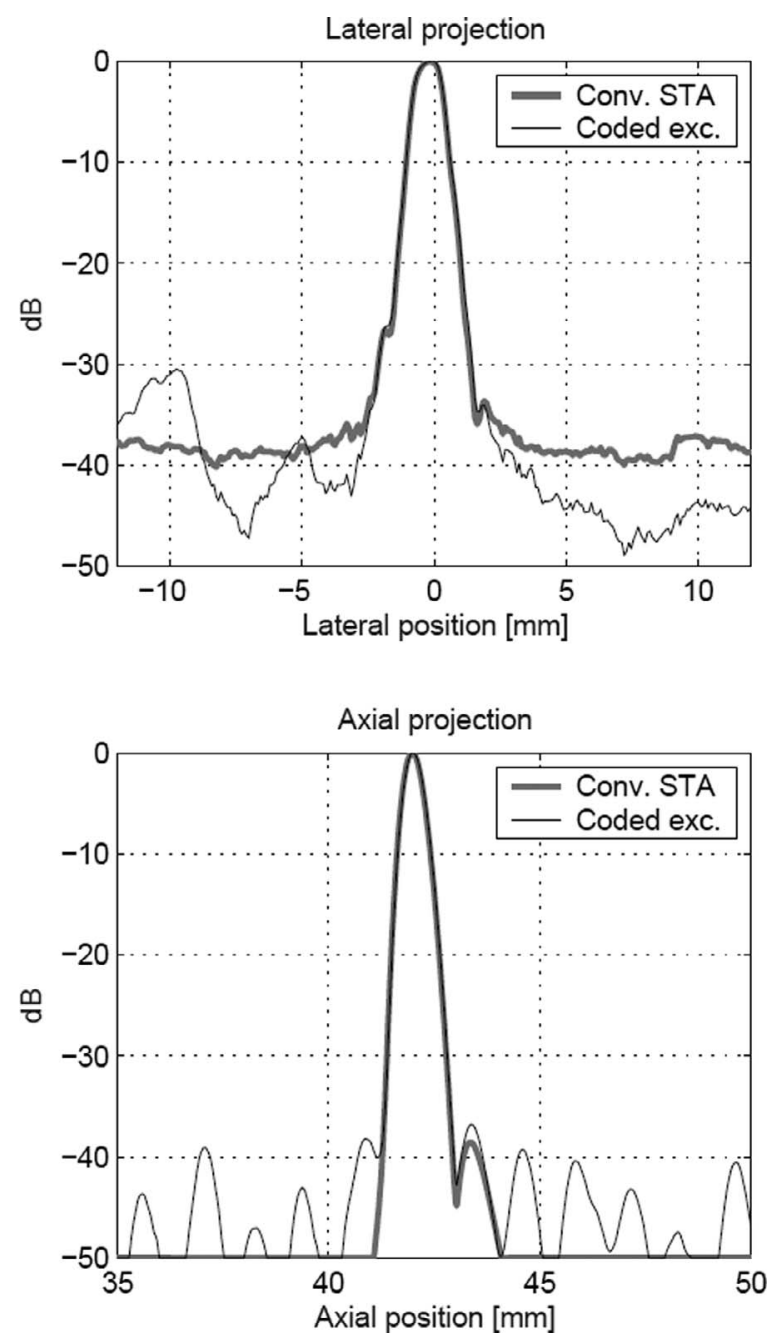

Fig. 13. The axial and lateral projections of the point spread functions in Fig. 12. The gray curve indicates the reference experiment, and the black solid curve represents the code division experiment. The axial sidelobe level of the code division experiment is approximately $-40 \mathrm{~dB}$. The sidelobes is a result of estimation errors and are due to modeling errors (nonlinearities) and noise. 
lation in which a gain in SNR was $\sim 1.5 \mathrm{~dB}$ compared to the pulsed case using a standard synthetic transmit aperture ultrasound imaging technique with twice the number of transmissions. The expected gain in SNR, however, was $\sim 12.5 \mathrm{~dB}$ and the discrepancy was traced to choice of code sequences. The PSFs at different depths also were simulated using Field II and were compared to conventional STA in which the performance was identical. The method was also tested in a physical system (RASMUS). The PSF was measured in a water tank and was compared to a setup using conventional STA. The performance was comparable to the reference experiment with slightly poorer axial contrast. It shall be noted that the method is based on a linear assumption, and even though water is a strongly nonlinear medium (because no attenuation is present), the method still performs acceptably. Future work will be focused on: code design (better cross correlation and autocorrelation properties), to achieve the theoretical SNR predicted in this paper, relaxing the demand on code duration, possibly by finding redundancies in the impulse response matrix $\mathbf{H}$, and exploiting this. To illustrate this, imagine a system with two transducer elements acting as both transmitters and receivers. The first transducer element transmits the waveform $x_{1}(n)$ and the second element transmits $x_{2}(n)$. The two received signals can be written

$$
\begin{aligned}
& y_{1}(n)=h_{11}(n) \star x_{1}(n)+h_{12}(n) \star x_{2}(n), \\
& y_{2}(n)=h_{21}(n) \star x_{1}(n)+h_{22}(n) \star x_{2}(n),
\end{aligned}
$$

using the same notation as previously. For simple sources and receivers, acoustic reciprocity dictate that the positions of the source and receiver are interchangeable [30]. Therefore:

$$
h_{21}(n)=h_{12}(n)
$$

and the number of parameters in the impulse response matrix $\mathbf{H}$ will be reduced. This symmetry can be extended readily to a system with an arbitrary number of sensors.

\section{REFERENCES}

[1] S. Bennett, D. K. Peterson, D. Corl, and G. S. Kino, "A real-time synthetic aperture digital acoustic imaging system," in Proc. 10th Int. Symp. Acoust. Imag., 1982, pp. 669-692.

[2] S. I. Nikolov, "Synthetic aperture tissue and flow ultrasound imaging," Ph.D. thesis, Ørsted•DTU, Technical University of Denmark, Lyngby, Denmark, 2001.

[3] M. O'Donnell and L. J. Thomas, "Efficient synthetic aperture imaging from a circular aperture with possible application to catheter-based imaging," IEEE Trans. Ultrason., Ferroelect., Freq. Contr., vol. 39, pp. 366-380, 1992.

[4] M. Karaman, P. C. Li, and M. O'Donnell, "Synthetic aperture imaging for small scale systems," IEEE Trans. Ultrason., Ferroelect., Freq. Contr., vol. 42, pp. 429-442, 1995.

[5] C. Passmann and H. Ermert, "A 100-MHz ultrasound imaging system for dermatologic and ophthalmologic diagnostics," IEEE Trans. Ultrason., Ferroelect., Freq. Contr., vol. 43, pp. 545-552, 1996.

[6] C. H. Frazier and W. D. O'Brien, "Synthetic aperture techniques with a virtual source element," IEEE Trans. Ultrason., Ferroelect., Freq. Contr., vol. 45, pp. 196-207, 1998.
[7] M. O'Donnell, "Coded excitation system for improving the penetration of real-time phased-array imaging systems," IEEE Trans. Ultrason., Ferroelect., Freq. Contr., vol. 39, pp. 341-351, 1992.

[8] T. X. Misaridis, K. Gammelmark, C. H. Jørgensen, N. Lindberg, A. H. Thomsen, M. H. Pedersen, and J. A. Jensen, "Potential of coded excitation in medical ultrasound imaging," Ultrasonics, vol. 38, pp. 183-189, 2000.

[9] T. X. Misaridis, M. H. Pedersen, and J. A. Jensen, "Clinical use and evaluation of coded excitation in B-mode images," in Proc. IEEE Ultrason. Symp., 2000, pp. 1689-1693.

[10] T. X. Misaridis and J. A. Jensen, "An effective coded excitation scheme based on a predistorted FM signal and an optimized digital filter," in Proc. IEEE Ultrason. Symp., 1999, pp. 15891593.

[11] T. Misaridis, "Ultrasound imaging using coded signals," Ph.D. thesis, Ørsted•DTU, Technical University of Denmark, Lyngby, Denmark, 2001.

[12] K. L. Gammelmark and J. A. Jensen, "Multielement synthetic transmit aperture imaging using temporal encoding," IEEE Trans. Med. Imag., vol. 22, no. 4, pp. 552-563, 2003.

[13] R. Y. Chiao, L. J. Thomas, and S. D. Silverstein, "Sparse array imaging with spatially-encoded transmits," in Proc. IEEE Ultrason. Symp., 1997, pp. 1679-1682.

[14] T. Misaridis and J. A. Jensen, "Space-time encoding for high frame rate ultrasound imaging," Ultrasonics, vol. 40, pp. 593$597,2002$.

[15] R. Y. Chiao and L. J. Thomas, "Synthetic transmit aperture using orthogonal golay coded excitation," in Proc. IEEE Ultrason. Symp., 2000, pp. 1469-1472.

[16] F. Gran and J. A. Jensen, "Multi element synthetic aperture transmission using a frequency division approach," in Proc. IEEE Ultrason. Symp., 2003, pp. 1942-1946.

[17] F. Gran and J. A. Jensen, "Spatio-temporal encoding using narrow-band linearly frequency modulated signals in synthetic aperture ultrasound imaging," in Proc. SPIE-Progr. Biomed. Opt. Imag., 2005, pp. 405-416.

[18] F. Gran, J. A. Jensen, and A. Jakobsson, "A code division technique for multiple element synthetic aperture transmission," in Proc. SPIE-Progr. Biomed. Opt. Imag., 2004, pp. 300-306.

[19] F. Gran and J. A. Jensen, "Identification of spatial impulse responses for multi source transmission," in Proc. Thirty-Eighth Annular Asilomar Conference on Signals, Systems, and Computers, 2004, pp. 168-172.

[20] J. Shen and E. S. Ebbini, "A new coded-excitation ultrasound imaging system-Part 1: Basic principles," IEEE Trans. Ultrason., Ferroelect., Freq. Contr., vol. 43, no. 1, pp. 131-140, 1996.

[21] J. Shen and E. S. Ebbini, "A new coded-excitation ultrasound imaging system-Part 2: Operator design," IEEE Trans. Ultrason., Ferroelect., Freq. Contr., vol. 43, no. 1, pp. 141-148, 1996.

[22] J. A. Jensen, "A model for the propagation and scattering of ultrasound in tissue," J. Acoust. Soc. Amer., vol. 89, pp. 182$191,1991$.

[23] J. A. Jensen, Estimation of Blood Velocities Using Ultrasound: A Signal Processing Approach. New York: Cambridge Univ. Press, 1996.

[24] L. Ljung, System Identification, Theory for the User. Englewood Cliffs, NJ: Prentice-Hall, 1987.

[25] S. H. Friedberg, A. J. Insel, and L. E. Spence, Linear Algebra. Prentice-Hall International Editions, 1992.

[26] G. Kang, E. Kosta, M. Weckerle, and E. Schulz, "Optimum channel estimation over frequency-selective fading channel in multiple antenna systems," in Proc. Commun. Technol. ICCT, 2003, pp. $1799-1803$.

[27] J. A. Jensen and N. B. Svendsen, "Calculation of pressure fields from arbitrarily shaped, apodized, and excited ultrasound transducers," IEEE Trans. Ultrason., Ferroelect., Freq. Contr., vol. 39, pp. 262-267, 1992.

[28] J. A. Jensen, "Field: A program for simulating ultrasound systems," 10th Nordic-Baltic Conf. Biomed. Imag., vol. 34, 1996, pp. 351-353.

[29] J. A. Jensen, O. Holm, L. J. Jensen, H. Bendsen, H. M. Pedersen, K. Salomonsen, J. Hansen, and S. Nikolov, "Experimental ultrasound system for real-time synthetic imaging," in Proc. IEEE Ultrason. Symp., 1999, pp. 1595-1599.

[30] L. E. Kinsler, A. R. Frey, A. B. Coppens, and J. V. Sanders, Fundamentals of Acoustics. 3rd ed. New York: Wiley, 1982. 


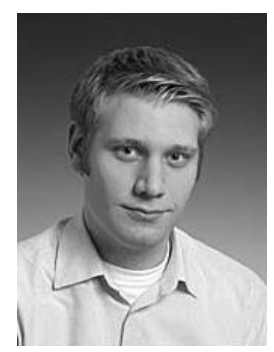

Fredrik Gran earned his M.Sc. degree in engineering physics from Lund University, Sweden, in 2002 and the Ph.D. degree in electrical engineering from the Technical University of Denmark, Denmark, in 2005. He is currently a postdoctoral researcher at the Center for Fast Ultrasound Imaging at the Technical University of Denmark.

His research interests include spatial and temporal encoding in medical ultrasound and signal processing.

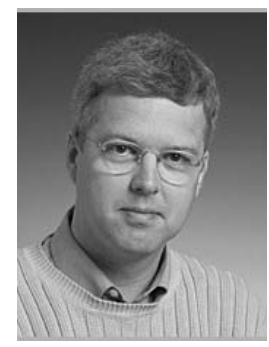

Jørgen Arendt Jensen (M'93-SM'02) earned his M.S. degree in electrical engineering in 1985 and the Ph.D. degree in 1989 both from the Technical University of Denmark. He received the Dr.Techn. degree from the university in 1996. He has published more than 140 journal and conference papers on signal processing and medical ultrasound and the book, Estimation of Blood Velocities Using Ultrasound, Cambridge University Press, in 1996. He is also developer of the Field II simulation program. He has been a visiting scientist at Duke University, Stanford University, and the University of Illinois at UrbanaChampaign. He is currently full professor of Biomedical Signal Processing at the Technical University of Denmark at Ørsted•DTU and head of Center for Fast Ultrasound Imaging. He is also adjunct full professor at the Faculty of Health Sciences at the University of Copenhagen. He has given courses on blood velocity estimation at both Duke University and the University of Illinois and teaches biomedical signal processing and medical imaging at the Technical University of Denmark. He has given several short courses on simulation, synthetic aperture imaging, and flow estimation at international scientific conferences. He has received several awards for his research. He is also the co-organizer of a new biomedical engineering education program offered by the Technical University of Denmark and the University of Copenhagen. His research is centered around simulation of ultrasound imaging, synthetic aperture imaging and blood flow estimation, and constructing systems for such imaging. 